\title{
Retraction Note to: Diagnostic procedures for improving of the KIT (CD117) expressed allele burden for the liver metastases from uterus mast cell tumors: prognostic value of the metastatic pattern and tumor biology
}

\author{
Ehsan Hosseini ${ }^{1}$ - Behnam Pedram ${ }^{2}$ - Ali Mohammad Bahrami ${ }^{1}$ - Seyed Rashid Touni ${ }^{3}$. \\ Hamed Zamankhan Malayeri ${ }^{4}$ - Aram Mokarizadeh ${ }^{5}$ • Mehdi Pourzaer ${ }^{6}$. \\ Maryam Pourzaer $^{7} \cdot$ Shahram Zehtabian $^{8} \cdot$ Sheida Mohajer $^{9} \cdot$ Sharareh Ahmadi $^{10}$
}

Published online: 5 November 2016

(C) International Society of Oncology and BioMarkers (ISOBM) 2016

\section{OI 10.1007/s13277-014-2666-6}

This article has been retracted at the request of the Editor-in-Chief, the International Society of Oncology and BioMarkers (ISOBM) and the Publisher per the Committee on Publication Ethics guidelines. The article shows evidence of irregularities in authorship during the submission process, there is strong reason to believe that the peer review process was compromised and the article contains patchwork plagiarism from a variety of sources.

The main sources are (amongst others):

Ehsan Hosseini, Behnam Pedram, Ali Mohammad BahramiEmail author, Mohammad Hossein Jaberi

The online version of the original article can be found at $\mathrm{http} / / \mathrm{dx}$.doi. org/10.1007/s13277-014-2666-6.

Ali Mohammad Bahrami

am.bahrami@ilam.ac.ir

$\triangle$ Aram Mokarizadeh

A.mokarizadeh@muk.ac.ir

1 Faculty of Para Veterinary Medicine, Ilam University, Ilam, Iran

2 Department of Pathobiology, Susangerd Branch, Islamic Azad University, Susangerd, Iran

3 Department of Basic Science, Faculty of Veterinary Medicine, Urmia University, Urmia, Iran

4 Department of Internal Medicine, Faculty of Veterinary Medicine, Tehran University, Tehran, Iran
Moghaddam, Javad Javanbakht, Fatemeh Emami Ghomi, Najme Jaberi Moghaddam, Mobin Koohestani and Radmehr Shafiee, Cutaneous mast cell tumor (Mastocytoma): Cyto- histopathological and haematological investigations. Tumor Biol. 2014; 9:9 DOI: 10.1186/1746-1596-9-9

Catherine G. Lamm, Adam W. Stern, Amanda J. Smith, Emily J. Cooper, Steven W. Ullom, Gregory A. Campbell, Disseminated Cutaneous Mast Cell Tumors with Epitheliotropism and Systemic Mastocytosis in a Domestic Cat, Journal of Veterinary Diagnostic Investigation. 2009; 21:5 710-715 DOI: $10.1177 / 104063870902100520$

Tzu-Yin Lin, Alexander Hamberg, Rebecca Pentecost, Maxey Wellman, Paul Stromberg, Mast Cell Tumors in a Llama (Lama Glama), Journal of Veterinary Diagnostic

5 Department of Immunology, Faculty of Medicine, and Cellular and Molecular Research Center, Kurdistan University of Medical Sciences, Sanandaj, Iran

6 Faculty of Veterinary Science, Karaj Branch, Islamic Azad University, Alborz, Iran

7 Department of Biology, Faculty of Basic Science, Damghan Branch, Islamic Azad University, Damghan, Iran

8 Department of Microbiology, Faculty of Medicine, Kermanshah University of Medical Sciences, Kermanshah, Iran

9 Chronic Respiratory Disease Research Center, National Research Institue of Tuberculosis and Lung Diseases, Massih Daneshvary Hospital, Shahid Beheshti University of Medicine, Tehran, Iran

10 Faculty of Medicine, Kermanshah University of Medical Sciences, Kermanshah, Iran 
Investigation $2010 ; 22: 5$ 808-811 DOI: 10.1177 $/ 104063871002200531$

Mery Giantin, Marta Vascellari, Emanuela Maria Morello, Katia Capello, Antonella Vercelli, Anna Granato Rosa Maria Lopparelli, Chiara Nassuato, Antonio Carminato, Marina Martano, Franco Mutinelli, Mauro Dacasto, c-KIT messenger RNA and protein expression and mutations in canine cutaneous mast cell tumors correlations with post-surgical prognosis, Journal of Veterinary Diagnostic Investigation January, 2012; 24:1 116-126 doi:10.1177/1040638711425945

M. Giantin, L. Aresu, S. Benali, A. Aricò, E.M. Morello, M. Martano, M. Vascellari, M. Castagnaro, R.M. Lopparelli, V. Zancanella, A. Granato, F. Mutinelli, M. Dacasto, Expression of Matrix Metalloproteinases, Tissue Inhibitors of Metalloproteinases and Vascular Endothelial Growth Factor in Canine Mast Cell Tumours, Journal of Comparative Pathology. 2012; 147:4 419-429 DOI: 10.1016 /j.jcpa.2012.01.011

In addition, figure 9 "Uterus: canine, mast cell tumor. Diffuse cytoplasmic KIT localization and cells expressing KIT are identified by blue labeling. The majority of neoplastic mast cells express KIT marker within the cytoplasm, often obscuring the nucleus. $\times 400$ magnification" and figure 10 "Fine-needle aspirate smear of a uterus mass from the mast cell tumor of a dog. There are many large round cells with centrally placed round nuclei, clear cytoplasm, pleomorphism, and poorly differentiated mast cells" are duplicating:

13048_2014_105_MOESM9_ESM.png Authors' original file for figure 9 (online resource)

13048_2014_105_MOESM10_ESM.png Authors' original file for figure 10 (online resource)

Published in:

Ali Mohammad Bahrami, Fariba Khaki, Shahram Zehtabian, Javad Cheraghi, Mehdi Rashnavadi, Mohammad Reza Hafezi Ahmadi, Mostafa Naderafif, Soheil Javaherypour, Siamak Mohsenzadeh, Ehsan Hosseini, Hamed Masoudi, Mehdi Pourzaer, Uterine mast cell tumor: a clinical and cytohistopathological study, Journal of Ovarian Research 2014; 7:105 DOI: 10.1186/s13048-014-0105-3 http://ovarianresearch.biomedcentral.com/articles/10.1186 /s13048-014-0105-3 (online resource)

As such the validity of the content of this article cannot be verified. 